\title{
Desingularised method of double layer fundamental solutions for potential flow problems
}

\author{
B. Šarler \\ Laboratory for Multiphase Processes, \\ University of Nova Gorica, Slovenia
}

\begin{abstract}
This paper describes an application of the recently proposed Modified Method of Fundamental Solutions (MMFS) to potential flow problems. The solution in two dimensional Cartesian coordinates is represented in terms of the double layer fundamental solution of the Laplace equation. The collocation is used for determination of the expansion coefficients. This novel method does not require a fictitious boundary unlike the conventional Method of Fundamental Solutions (MFS). The source and collocation points thus coincide on the physical boundary of the system. The desingularised value of the fundamental solution is in case of the coincidence of the collocation and source points determined from the respective integral equations logic. The values of the derivatives of the double layer fundamental solution in the coordinate directions, as required in potential flow calculations, are calculated indirectly from the considerations of the constant potential field. A numerical example of potential flow around the two dimensional circular region is shown. The results with the MMFS are compared with the results of the classical single layer MFS with an artificial boundary, and the analytical solution. It is shown that the MMFS gives better accuracy of the velocity components as compared with the classical MFS.
\end{abstract}

Keywords: potential flow, method of fundamental solutions, desingularisation, double layer potential.

\section{Introduction}

In recent years there has been strong development in mesh reduction methods in which the polygon-like meshes are reduced or avoided. The MFS is a 
numerical technique that falls into the class of methods generally called boundary methods. The other well known representative of these methods is the Boundary Element Method (BEM) [1]. Both methods are best applicable in situations where a fundamental solution to the partial differential equation in question is known. In such cases, the dimensionality of the discretization is reduced. BEM for example requires polygonisation of the boundary surfaces in general 3D cases, and boundary curves in general 2D cases. This method requires solution of the complicated regular, weakly singular, strongly singular, and hypersingular integrals over boundary segments, which is usually a cumbersome and non-trivial task. The MFS has certain advantages over BEM, which are mostly visible in the fact that only pointisation of the boundary is needed, completely avoiding any integral evaluations, and making no principal difference in coding between the $2 \mathrm{D}$ and the $3 \mathrm{D}$ cases. A comprehensive survey of the MFS and related methods for elliptic boundary value problems can be found in [2-4]. In the present paper, the potential flow problem, previously solved by the least squares version of MFS [5] and collocation version of MFS [6] is solved by the MMFS. This novel method, which essentially represents a sort of blend between BEM and MFS, was originally developed by Young et al. [7] using collocation with the double layer Laplace equation fundamental solution. The method has been further extended to the single-layer Laplace equation fundamental solution in [8]. The main drawback of the MFS is representation of positions of the source points that need to be positioned outside the boundary. In cases where they are too close to the boundary, the solution is not accurate. In cases where they are too far away from the boundary, the discretisation matrix becomes ill conditioned. The novel MMFS overcomes this difficulty by allowing the source point positions to coincide with the collocation points on the physical boundary. A desingularisation technique thus has to be employed in order to be able to allow bounded values in the discretisation matrix. The desingularisation has been derived through the properties of the double layer potential in [7] and through the indirect BEM formulation in [8]. In the present paper, the desingularisation is extended to the calculation of the desingularised values through the direct BEM approach, as well as calculation of the desingularised value of the partial (not normal) derivatives on the boundary, which was not the case in the previous two cited MMFS pioneering papers by D. L. Young's group.

\section{Governing equations}

Consider a connected two-dimensional domain $\Omega$ with boundary $\Gamma$. The domain is filled by a fluid that undergoes potential flow. The boundary is divided into the part $\Gamma^{E}$, which represents the external boundaries of the system and into the part $\Gamma^{I}$, which represents the internal boundary of the system i.e. $\Gamma=\Gamma^{E} \cup \Gamma^{I}$. The potential $\Phi$ is governed by the following boundary value problem: Laplace equation

$$
\nabla^{2} \Phi=0
$$


and boundary conditions of the Dirichlet and Neumann type, located at the Dirichlet $\Gamma^{D}$ and Neumann $\Gamma^{N}$ parts of the boundary $\Gamma$, i.e. $\Gamma=\Gamma^{D} \cup \Gamma^{N}$

$$
\Phi(\mathbf{p})=\bar{\Phi}^{D}(\mathbf{p}), \quad \mathbf{p} \in \Gamma^{D}, \quad \frac{\partial \Phi}{\partial \mathbf{n}_{\Gamma}}(\mathbf{p})=\bar{\Phi}^{N}(\mathbf{p}), \quad \mathbf{p} \in \Gamma^{N},
$$

with $\mathbf{p}$ standing for the position vector and $\mathbf{n}_{\Gamma}$ for the outward normal on the boundary $\Gamma \cdot \bar{\Phi}^{D}$ and $\bar{\Phi}^{N}$ representing the Dirichlet and Neumann boundary condition forcing functions. Let us introduce a two dimensional Cartesian coordinate system with ortho-normal base vectors $\mathbf{i}_{x}$ and $\mathbf{i}_{y}$ and coordinates $p_{x}$ and $p_{y}$, i.e. $\mathbf{p}=p_{x} \mathbf{i}_{x}+p_{y} \mathbf{i}_{y}$. The potential field velocity components are calculated from the potential $\Phi$ as

$$
v_{\xi}(\mathbf{p})=\frac{\partial \Phi}{\partial p_{\xi}}(\mathbf{p}) ; \xi=x, y .
$$

It is the purpose of this paper to determine the steady state potential flow components as a function of the posed geometry, governing equation and boundary conditions.

\section{Solution procedure}

\subsection{Solution of the potential flow}

The common points of the MFS and MMFS for solution of the potential flow field are elaborated first. The differences are elaborated afterwards. The solution of the potential $\Phi$ is represented by the $N_{\Gamma}$ global approximation functions $\psi_{n}(\mathbf{p})$ and their coefficients $\alpha_{n}$

$$
\Phi(\mathbf{p}) \approx \sum_{n=1}^{N_{\Gamma}} \psi_{n}(\mathbf{p}) \alpha_{n}
$$

The global approximation functions have the property

$$
\nabla^{2} \psi_{n}(\mathbf{p})=\left\{\begin{array}{rl}
0 ; \mathbf{p} & \neq \mathbf{p}_{n} \\
\delta\left(\mathbf{p}_{n}\right) ; \mathbf{p}=\mathbf{p}_{n}
\end{array} ; n=1,2, \ldots, N_{\Gamma},\right.
$$

i.e., they are fundamental solutions of the Laplace operator. $\delta$ denotes the Kronecker symbol. For two dimensional problems in Cartesian coordinates, the fundamental solution equals to

$$
\psi_{n}^{*}(\mathbf{p})=\frac{1}{2 \pi} \log \frac{r^{*}}{r_{n}} ; r_{n}^{2}=\mathbf{r}_{n} \cdot \mathbf{r}_{n}, \mathbf{r}_{n}=\mathbf{p}-\mathbf{s}_{n}=\left(p_{x}-s_{n x}\right) \mathbf{i}_{x}+\left(p_{y}-s_{n y}\right) \mathbf{i}_{y},
$$

where $r^{*}$ denotes the reference radius and $p_{0 x}$ and $p_{0 y}$ represent the mean coordinates of the $\Gamma \cup \Omega$. The double layer fundamental solution, defined by

$$
\psi_{n}^{\diamond}(\mathbf{p})=\frac{\partial \psi^{*}}{\partial \mathbf{n}_{s}}(\mathbf{p})=\frac{1}{2 \pi}\left[\frac{\left(p_{x}-s_{n x}\right) n_{s x}}{\left(p_{x}-s_{n x}\right)^{2}+\left(p_{y}-s_{n y}\right)^{2}}+\frac{\left(p_{y}-s_{n y}\right) n_{s y}}{\left(p_{x}-s_{n x}\right)^{2}+\left(p_{y}-s_{n y}\right)^{2}}\right]
$$


is used in the present paper. Let us introduce the boundary condition indicators in order to be able to represent the boundary collocation equations in a compact form. The Dirichlet $\chi^{D}$ and Neumann $\chi^{N}$ type of boundary conditions indicators are

$$
\chi^{D}(\mathbf{p})=\left\{\begin{array}{c}
1 ; \mathbf{p} \in \Gamma^{D} \\
0 ; \mathbf{p} \notin \Gamma^{D}
\end{array}, \quad \chi^{N}(\mathbf{p})=\left\{\begin{array}{c}
1 ; \mathbf{p} \in \Gamma^{N} \\
0 ; \mathbf{p} \notin \Gamma^{N}
\end{array} .\right.\right.
$$

The coefficients are calculated from a system of $N_{\Gamma}$ algebraic equations

$$
\begin{gathered}
\sum_{n=1}^{N_{\Gamma}} \Psi_{j n} \alpha_{n}=b_{j} ; j=1,2, \ldots, N_{\Gamma} . \\
\Psi_{j n}=\chi^{D}\left(\mathbf{p}_{j}\right) \psi_{n}\left(\mathbf{p}_{j}\right)+\chi^{N}\left(\mathbf{p}_{j}\right) \frac{\partial \psi_{n}}{\partial \mathbf{n}_{\Gamma}}\left(\mathbf{p}_{j}\right), \\
b_{j}=\chi^{D}\left(\mathbf{p}_{j}\right) \bar{\Phi}^{D}\left(\mathbf{p}_{j}\right)+\chi^{N}\left(\mathbf{p}_{j}\right) \frac{\partial \bar{\Phi}^{N}}{\partial \mathbf{n}_{\Gamma}}\left(\mathbf{p}_{j}\right),
\end{gathered}
$$

The coefficients $\alpha_{n}$ can be expressed through inversion of the system (10), which gives

$$
\alpha_{n}=\sum_{j=1}^{N_{\Gamma}} \Psi_{n j}^{-1}\left[\chi^{D}\left(\mathbf{p}_{j}\right) \bar{\Phi}^{D}\left(\mathbf{p}_{j}\right)+\chi^{N}\left(\mathbf{p}_{j}\right) \bar{\Phi}^{N}\left(\mathbf{p}_{j}\right)\right] ; \quad n=1,2, \ldots, N_{\Gamma} .
$$

The velocity field components are calculated as

$$
v_{\xi}(\mathbf{p})=\sum_{j=1}^{N_{\Gamma}} \frac{\partial \psi_{n}}{\partial p_{\xi}}(\mathbf{p}) \alpha_{n} ; \xi=x, y .
$$

\subsection{Classical method of fundamental solutions}

The fundamental solution source points are located outside physical boundary, i.e. $\mathbf{p}_{j} \neq \mathbf{s}_{j}$ and $\mathbf{s}_{j} \notin \Omega$ in the classical MFS. One can consider that they form an artificial boundary. The proper location of the source points is not a trivial task. It can be observed that the accuracy improves with the increasing distance from the physical boundary up to some extent. However, the collocation matrices become increasingly ill conditioned with increased distance from the boundary. The explicit form of the partial derivatives of the fundamental solution are

$$
\begin{aligned}
& \frac{\partial \psi^{\diamond}}{\partial p_{x}}(\mathbf{p})=-\frac{1}{\pi} \frac{\left(p_{x}-s_{n x}\right)\left(p_{y}-s_{n y}\right) n_{s y}}{\left[\left(p_{x}-s_{n x}\right)^{2}+\left(p_{y}-s_{n y}\right)^{2}\right]^{2}} \\
& +\frac{1}{2 \pi}\left[\frac{1}{\left(p_{x}-s_{n x}\right)^{2}+\left(p_{y}-s_{n y}\right)^{2}}-\frac{2\left(p_{x}-s_{n x}\right)\left(p_{x}-s_{n x}\right)}{\left[\left(p_{x}-s_{n x}\right)^{2}+\left(p_{y}-s_{n y}\right)^{2}\right]^{2}}\right] n_{s x}
\end{aligned}
$$




$$
\begin{aligned}
& \frac{\partial \psi^{\diamond}}{\partial p_{y}}(\mathbf{p})=-\frac{1}{\pi} \frac{\left(p_{y}-s_{n y}\right)\left(p_{x}-s_{n x}\right) n_{s y}}{\left[\left(p_{x}-s_{n x}\right)^{2}+\left(p_{y}-s_{n y}\right)^{2}\right]^{2}} \\
& +\frac{1}{2 \pi}\left[\frac{1}{\left(p_{x}-s_{n x}\right)^{2}+\left(p_{y}-s_{n y}\right)^{2}}-\frac{2\left(p_{y}-s_{n y}\right)\left(p_{y}-s_{n y}\right)}{\left[\left(p_{x}-s_{n x}\right)^{2}+\left(p_{y}-s_{n y}\right)^{2}\right]^{2}}\right] n_{s x}
\end{aligned}
$$

\subsection{Modified method of fundamental solutions}

The key point of the modified method of fundamental solutions represents desingularisation of the value of the fundamental solution, because in this case the source and the collocation points coincide, i.e. $\mathbf{p}_{j}=\mathbf{s}_{j}$. The desingularisation value can be directly set from the

$$
\int_{\Gamma} \Phi \nabla \psi^{*} \cdot d \boldsymbol{\Gamma}-\int_{\Gamma} \psi^{*} \nabla \Phi \cdot d \boldsymbol{\Gamma}+c(\mathbf{s}) \Phi(\mathbf{s})=0 .
$$

The derivation of the upper equation with respect to $\mathbf{n}_{s}$ gives

$$
\int_{\Gamma} \Phi \nabla \psi^{\diamond} \cdot d \boldsymbol{\Gamma}-\int_{\Gamma} \psi^{\diamond} \nabla \Phi \cdot d \boldsymbol{\Gamma}+c(\mathbf{s}) \frac{\partial \Phi}{\partial \mathbf{n}_{s}}(\mathbf{s})=0 .
$$

In case of the constant potential $\Phi$, the equation (18) reduces to

$$
\int_{\Gamma} \Phi \nabla \psi^{*} \cdot d \boldsymbol{\Gamma}+c(\mathbf{s}) \Phi(\mathbf{s})=-\int_{\Gamma} \Phi \psi^{\diamond} d \Gamma+c(\mathbf{s}) \Phi(\mathbf{s})=0,
$$

and the equation (19) into

$$
\int_{\Gamma} \nabla \psi^{\diamond} d \boldsymbol{\Gamma}=0
$$

The calculation of the desingularised value of the double layer fundamental solution can be performed through the discretisation of the Equation (20) in the following simple way

$$
\begin{gathered}
-\sum_{n=1}^{N_{\Gamma}} \frac{\ell_{n-}+\ell_{n+}}{2} \psi_{j}^{\diamond}\left(\mathbf{p}_{n}\right)+c_{s}\left(\mathbf{s}_{j}\right)=0, \\
\psi_{j}^{\diamond}\left(\mathbf{p}_{j}\right)=\frac{1}{\ell_{j-}+\ell_{j+}}\left[1-\sum_{\substack{n=1 \\
n \neq j}}^{N_{\Gamma}}\left(\ell_{n-}+\ell_{n+}\right) \psi_{j}^{\diamond}\left(\mathbf{p}_{n}\right)\right] .
\end{gathered}
$$

The calculation of the desingularised value of the double layer fundamental solution normal derivative can be performed through the discretisation of the Equation (21) in the following simple way

$$
\begin{gathered}
\sum_{n=1}^{N_{\Gamma}} \frac{\ell_{n-}+\ell_{n+}}{2} \frac{\partial \psi_{n}^{\diamond}}{\partial \mathbf{n}_{\Gamma}}\left(\mathbf{p}_{j}\right)=0, \\
\frac{\partial \psi_{j}^{\diamond}}{\partial \mathbf{n}_{\Gamma}}\left(\mathbf{p}_{j}\right)=-\frac{1}{\ell_{j-}+\ell_{j+}}\left[\sum_{\substack{n=1 \\
n \neq j}}^{N_{\Gamma}}\left(\ell_{n-}+\ell_{n+}\right) \frac{\partial \psi_{j}^{\diamond}}{\partial \mathbf{n}_{\Gamma}}\left(\mathbf{p}_{j}\right)\right] .
\end{gathered}
$$


The internal and external boundaries are given by a vector of points $\mathbf{p}_{k} ; k=1,2, \ldots, N_{\Gamma}^{B} ; B=I, E$. The length $\ell_{k}$ of the contour between the boundary points $\mathbf{p}_{k}$ and $\mathbf{p}_{k-1}$ is parametrised by the simple Euclidean distance

$$
\ell_{k \pm}=\left[\left(p_{k x}-p_{k \pm 1 x}\right)^{2}+\left(p_{k y}-p_{k \pm 1 y}\right)^{2}\right]^{1 / 2},
$$

with the cyclic index conditions $k-1=N_{\Gamma}^{B} ; k=1, k+1=1 ; k=N_{\Gamma}^{B}$. The total Euclidean length $\ell_{\Gamma}$ of the boundary contour equals to

$$
\ell_{\Gamma}=\sum_{k=1}^{N_{\Gamma}^{B}} \ell_{k} .
$$

The derivatives of the fundamental solution can be calculated in the following indirect way. Let us assume a pure Dirichlet problem with all the boundary values set to a constant $\bar{\Phi}^{D}(\mathbf{p})=c ; \mathbf{p} \in \Gamma$. We obtain in this case

$$
\begin{gathered}
\Phi\left(\mathbf{p}_{j}\right)=c=\sum_{n=1}^{N_{\Gamma}} \psi_{n}\left(\mathbf{p}_{j}\right) \alpha_{n}^{c}, \\
\frac{\partial}{\partial p_{\xi}} \Phi\left(\mathbf{p}_{j}\right)=\sum_{n=1}^{N_{\Gamma}} \frac{\partial}{\partial p_{\xi}} \psi_{n}\left(\mathbf{p}_{j}\right) \alpha_{n}^{c}=0 ; \xi=x, y .
\end{gathered}
$$

The desingularised value of the partial derivative can be calculated as

$$
\frac{\partial}{\partial p_{\xi}} \psi_{j}^{\diamond}\left(\mathbf{p}_{j}\right)=-\frac{1}{\alpha_{j}^{c}} \sum_{\substack{n=1 \\ n \neq j}}^{N_{\Gamma}} \frac{\partial}{\partial p_{\xi}} \psi_{n}^{\diamond}\left(\mathbf{p}_{j}\right) \alpha_{n}^{c} ; \xi=x, y .
$$

The desingularised value of the normal derivative can be calculated from the desingularised values of the partial derivatives as

$$
\frac{\partial \psi_{j}^{\diamond}}{\partial \mathbf{n}_{\Gamma}}\left(\mathbf{p}_{j}\right)=\frac{\partial}{\partial p_{x}} \psi_{j}^{\diamond}\left(\mathbf{p}_{j}\right) n_{x}\left(\mathbf{p}_{j}\right)+\frac{\partial}{\partial p_{y}} \psi_{j}^{\diamond}\left(\mathbf{p}_{j}\right) n_{y}\left(\mathbf{p}_{j}\right) .
$$

This represents an alternative way how to arrive to Equation (25). The present approach does not require a direct integration, which distinguishes it from the approach with the single layer fundamental solution [9] where a direct BEM-like integration is needed.

\section{Numerical example}

Potential flow arround a circle is considered for a numerical example. The flow is confined to a square (exterior) region $\Gamma^{E}, p_{x}^{-} \leq p_{x} \leq p_{x}^{+}, p_{y}^{-} \leq p_{y} \leq p_{y}^{+}$with $p_{x}^{+}=-p_{x}^{-}=p_{0}, p_{y}^{+}=-p_{y}^{-}=p_{0}$. The Dirichlet boundary conditions are defined at the square boundaries as

$$
\bar{\Phi}^{D}\left(p_{x}, p_{y}\right)=v_{0} p_{y} ; p_{x}=p_{x}^{ \pm}, p_{y}=p_{y}^{ \pm} .
$$

The potential field, defined from the boundary conditions (32) gives the following solution for the velocity field

$$
v_{0 x}=0, \quad v_{0 y}=v_{0} .
$$


A circular hole $\Gamma^{I}$ (internal boundary) with the radius $r_{0}$, centered around point $\mathbf{p}_{c}$ with coordinates $p_{c x}=\left(p_{x}^{+}+p_{x}^{-}\right) / 2, p_{c y}=\left(p_{y}^{+}+p_{y}^{-}\right) / 2$ with the Neumann boundary conditions

$$
\bar{\Phi}^{N}\left[p_{x}\left(\Gamma^{I}\right), p_{y}\left(\Gamma^{I}\right)\right]=0 ; \mathbf{p} \in \Gamma^{I} .
$$

is inserted into the square. The solution of the potential field for $r_{0} \ll p_{0}$ equals

$$
\Phi_{\text {ana }}=v_{0 y}\left(p_{y}-p_{c y}\right)\left[1+\frac{r_{0}^{2}}{\left(p_{x}-p_{c x}\right)^{2}+\left(p_{y}-p_{c y}\right)^{2}}\right],
$$

with $v_{0 y}$ defined from equation (46). The respective analytical solution for the velocity field is

$$
\begin{gathered}
v_{\text {ana } x}=-v_{0 x} \frac{2 v_{0 y} r_{0}^{2}\left(p_{x}-p_{c x}\right)\left(p_{y}-p_{c y}\right)}{\left(p_{x}-p_{c x}\right)^{2}+\left(p_{y}-p_{c y}\right)^{2}} \\
v_{\text {ana } y}=v_{0 x}\left[1+\frac{r_{0}^{2}}{\left(p_{x}-p_{c x}\right)^{2}+\left(p_{y}-p_{c y}\right)^{2}}\right]-\frac{2 v_{0 y} r_{0}^{2}\left(p_{y}-p_{c y}\right)^{2}}{\left(p_{x}-p_{c x}\right)^{2}+\left(p_{y}-p_{c y}\right)^{2}}
\end{gathered}
$$

We set $r_{0}=0,1 \mathrm{~m}, p_{0}=0,5 \mathrm{~m}$ for geometry. The square sides are virtually divided into 50 equal length segments and the collocation points are put at each of the segment centres. The total number of discretisation points on the external boundary is set to $N^{E}=200$. The circle is discretised by five different discretisations $N^{I}=8,16,32,64,128$. The Root Mean Square (RMS) error of the MMFS solution is defined as

$$
\begin{gathered}
\Phi_{\mathrm{rms}}=\sum_{n=1}^{N^{I}}\left\{\frac{1}{N^{I}}\left[\Phi\left(\mathbf{p}_{n}\right)-\Phi_{\mathrm{ana}}\left(\mathbf{p}_{n}\right)\right]^{2}\right\}^{1 / 2}, \\
v_{\xi \mathrm{rms}}=\sum_{n=1}^{N^{I}}\left\{\frac{1}{N^{I}}\left[v_{\xi}\left(\mathbf{p}_{n}\right)-v_{\xi \mathrm{ana}}\left(\mathbf{p}_{n}\right)\right]^{2}\right\}^{1 / 2} ; \xi=x, y, \\
v_{\mathrm{rms}}=\left(v_{x \mathrm{rms}}^{2}+v_{y \mathrm{rms}}^{2}\right)^{1 / 2} .
\end{gathered}
$$

The RMS errors of the potential, velocity components, and absolute value of velocity are in case of MMFS given in Table 1 as a function of the discretisation density $N^{I}$. One can observe monotone convergence of the results with finer discretisation. A comparison with the results of the MFS (with artificial boundary), based on the single layer fundamental solution of the Laplace quation (the results are tabulated in [9]) shows, that the potential is in the case of MFS calculated more accurately as in the case of MMFS for $N^{I}=8,16$. The velocity components and absolute value of velocity are better predicted by the present MMFS than in the classical MFS in all attempted discretisations. 


\section{Conclusions}

In recent years, the MFS has proved to be an effective alternative to the boundary element methods in specific problems. Due to its advantages with respect to the simplicity of formulation and the fact that the distribution of the calculation nodes is truly meshless, the method is an ideal candidate for the moving and free boundary problems. Its main drawback represents the "artificial boundary issue". This issue has been in this work overcome through the MMFS concept, based on the double layer fundamental solution. The desingularisation of the potential has been in the present work made through the direct integral equation concept. The desingularisation of the spatial derivatives has been made in an indirect way through the constant potential field concept. Only the discretisation nodes on the real boundary are thus required. Both approaches differ from the previous two pioneering works on the subject $[7,8]$. In addition, this paper extends the MMFS to potential flow situations. The components of the flow field are calculated more precisely with the MMFS as with the MFS at all

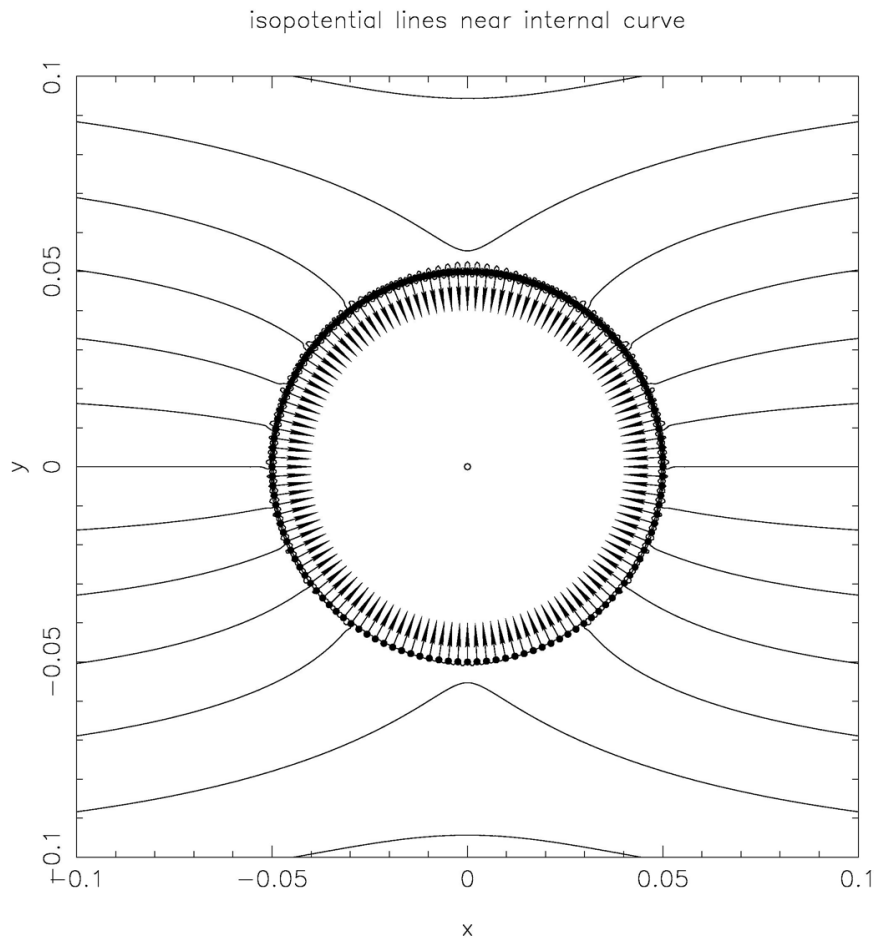

Figure 1: Calculated potential around the circle. The difference between the isopotential lines is $0,02 \mathrm{~m}^{2} / \mathrm{s}$. 
discretisations used. The presented developments can be straightforwardly upgraded to axisymmetric problems [10] by inclusion of the axisymmetric fundamental solution. The flow physics can be extended to Navier-Stokes flow by the strategy, proposed in [11] which uses the dual reciprocity with radial basis functions. The axisymmetric radial basis functions, such as thin plate splines [12] and multiquadrics [13] can be used for this purpose in axisymmetry.

Table 1: Calculated error of the solution as a function of the number of collocation points on the circle boundary.

\begin{tabular}{|r|l|l|l|l|}
\hline \multicolumn{1}{|r|}{$N^{I}$} & $\Phi_{\mathrm{rms}}$ & $v_{x \mathrm{rms}}$ & $v_{y \mathrm{rms}}$ & $v_{\mathrm{rms}}$ \\
\hline 8 & $1.037098 \mathrm{E}-02$ & $6.825820 \mathrm{E}-03$ & $1.177813 \mathrm{E}-02$ & $1.361309 \mathrm{E}-02$ \\
\hline 16 & $4.277747 \mathrm{E}-03$ & $6.385956 \mathrm{E}-03$ & $1.098282 \mathrm{E}-02$ & $1.270444 \mathrm{E}-02$ \\
\hline 32 & $1.704762 \mathrm{E}-03$ & $6.171696 \mathrm{E}-03$ & $1.063205 \mathrm{E}-02$ & $1.229351 \mathrm{E}-02$ \\
\hline 64 & $5.225166 \mathrm{E}-04$ & $6.069899 \mathrm{E}-03$ & $1.046481 \mathrm{E}-02$ & $1.209776 \mathrm{E}-02$ \\
\hline 128 & $4.806703 \mathrm{E}-05$ & $6.020260 \mathrm{E}-03$ & $1.038311 \mathrm{E}-02$ & $1.200219 \mathrm{E}-02$ \\
\hline
\end{tabular}

\section{Acknowledgement}

This paper forms a part of the project J2-0099: Multiscale Modelling and Simulation of Liquid-Solid Systems, sponsored by the Slovenian Research Agency. Financial support is kindly acknowledged.

\section{References}

[1] Wrobel LC, Aliabadi MH. The Boundary Element Method, J. Wiley, Chichester, 2002.

[2] Fairweather G, Karageorghis A. The method of fundamental solutions for elliptic boundary value problems, Advances in Computational Mathematics, 1998; 9: 69-95.

[3] Golberg MA, Chen CS. Discrete Projection Methods for Integral Equations, CMP, Southampton, 1997.

[4] Golberg MA, Chen CS. The method of fundamental solutions for potential, Helmholtz and diffusion problems, Boundary Integral Methods - Numerical and Mathematical Aspects, ed. Golberg, M. A., CMP, Southampton, 1998: 103-176.

[5] Johnston RL, Fairweather G. The method of fundamental solutions for problems in potential flow, Applied Mathematical Modelling, 1984; 8: 265270.

[6] Šarler B. Solution of a two-dimensional bubble shape in potential flow by the method of fundamental solutions, Engineering Analysis with Boundary Elements, 2006; 30: 227-235. 
[7] Young DL, Chen KH, Lee CW. Novel meshless method for solving the potential problems with arbitrary domain, Journal of Computational Physics, 2005; 209: 290-322.

[8] Young DL, Chen JT, Kao JH. A modified method of fundamental solutions with source on the boundary for solving Laplace equations with circular and arbitrary domains, CMES: Computer Modelling in Engineering and Sciences, 2007; 19: 197-221.

[9] Šarler B. Chapter 15: Modified method of fundamental solutions for potential flow problems. Chen CS, Karageorghis A, Smyrlis YS (Eds.), Method of Fundamental Solutions, Tech Science Press, Encinio, 2008; (to appear).

[10] Karageorghis A, Fairweather G. The method of fundamental solutions for axisymmetric potential problems, International Journal of Numerical Methods in Engineering, 1999; 44: 1653-1669.

[11] Šarler B. Towards a mesh-free solution of transport phenomena, Engineering Analysis with Boundary Elements, 2002; 26: 731-738.

[12] Šarler B. Axisymmetric augmented thin plate splines, Engineering Analysis with Boundary Elements, 1998; 21: 81-85.

[13] Šarler B, Jelić N, Kovačević I, Lakner M, Perko J. Axisymmetric multiquadrics, Engineering Analysis with Boundary Elements, 2006; 30: 137-142. 